Upsala J Med Sci 99: 315-338, 1994

\title{
7.2 Reference Intervals Based on a Danish Population
}

\author{
Ole Blaabjerg ${ }^{1}$, Inger Nørgaard ${ }^{2}$, Mogens Blom ${ }^{2}$, Jens Rahbek Nørgaard ${ }^{1}$, \\ Per Hyltoft Petersen ${ }^{1}$, Hanne Gry ${ }^{3}$, Adam Uldall ${ }^{4}$ \\ 1. Department of Clinical Chemistry, Odense University Hospital, DK-5000 Odense C, Denmark. \\ 2. Department of Clinical Chemistry, Hjørring Sygehus, DK-9800 Hjørring, Denmark. \\ 3. Medi-lab, Adelgade 5-7, DK-1304 Copenhagen, Denmark. \\ 4. Department of Clinical Chemistry, KAS Herlev, DK-2730 Herlev, Denmark.
}

\subsubsection{Need for Establishing Common Reference Intervals}

Is there a need for establishing common reference intervals for the quantities in clinical chemistry ? Yes, the reference intervals in current use are in principle established by each laboratory using a relevant number of healthy individuals and the present analytical technique. But, experience shows that it is very difficult to get documentation for any laboratory's reference intervals. The reason is that it is difficult and time consuming to do the work. So often reference intervals are based on an insufficient number of poorly characterized reference individuals or they are transferred from an old to a new analytical method when a new equipment, a new calibrator, or a new analytical principle is introduced in the laboratory - without the needed investigations. Thereby, the reference interval may remain unchanged whereas the analytical quality is drifting, or the traceability of the reference interval weakens considerably. Another approach is to use published reference intervals or to obtain them from other laboratories but, often without sufficient investigation of the transferability. The producer of some kits supply reference intervals together with the kit and this solution may be the best, as long as the producer can keep the stability of standardization and reagents.

The quality of calibration and of analytical procedure is a key-point when establishing reliable reference intervals as recently demonstrated by Frølich et al. (3). They compared results from fresh patient sera obtained in two laboratories in the same town using identical equipment and reporting identical reference intervals. Out of 15 analytes only four met the demands for sharing common reference intervals as defined by Gowans et al. (4). In an investigation Djurhuus et al. (2) demonstrated that for Danish laboratories the lower reference limit for S-Potassium varied from 3.2 to 3.7 $\mathrm{mmol} / \mathrm{L}$ whereas the results from an external analytical quality control varied by only $0.23 \mathrm{mmol} / \mathrm{L}$ for the same laboratories - indicating that the analytical technique is better than the estimated reference intervals. 
Concerning plasma proteins the poor standardization, due to insufficient traceability, of calibrators, is well known and it has therefore been accepted practice, that each laboratory should establish its own reference intervals. The reliability of these was investigated in Denmark in 1990 by an external control survey with an untreated liquid frozen serum pool collected from healthy subjects as control material. The results were compared to the reference intervals for each laboratory and it was anticipated that the result of the control pool should be located centrally in the interval.

\section{S-IgM}

Reference Intervals in Denmark (June 1990)

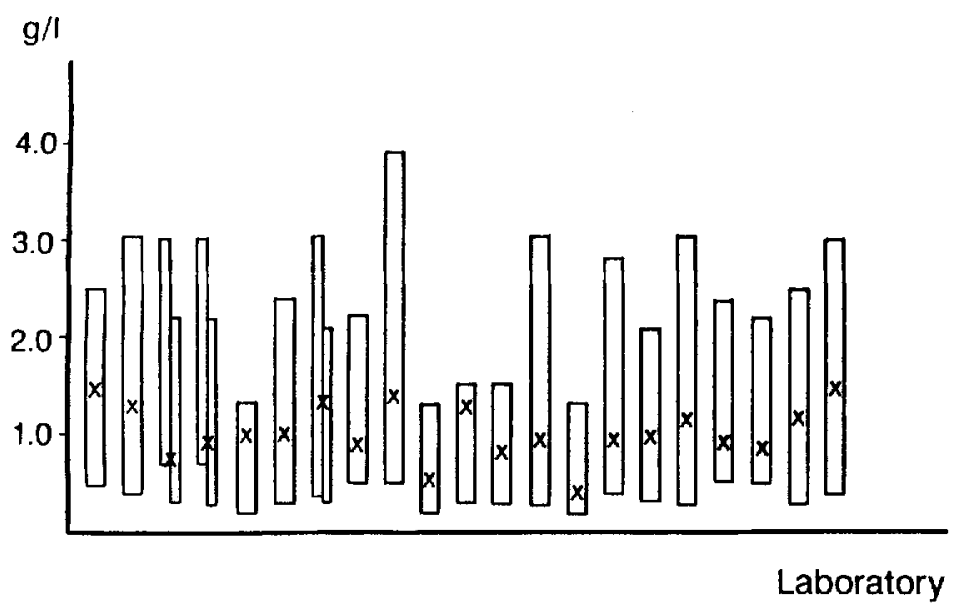

Fig. 7.2.1.1. Comparison of reference intervals and results from an external control survey in Denmark in 1990 for S-IgM. For each laboratory the reference interval is shown as a bar and the control result is indicated by a cross.

In Fig. 7.2.1.1 the results for S-IgM are illustrated. As the distribution of reference values is log-Gaussian the control result should be located just below the central value of the reference interval, and this seems to be the case for many of the laboratories. With a coefficient of variation, $\mathrm{CV}_{\mathrm{A}}$, of $28 \%$ for the control results, the figure thus illustrate that the reference intervals were established according to the standardization in each laboratory. The reference intervals, however, vary by a factor of three between the broadest and the most narrow, indicating insufficient number of reference individuals used for estimating the reference intervals. Three laboratories are reporting separate intervals for men and women. 
The results for S-Transferrin show a different pattern as illustrated in Fig. 7.2.1.2. In this case the $\mathrm{CV}_{\mathrm{A}}$ for the control is approx. $10 \%$, but the reference intervals are varying considerably, indicating a lack of traceability for most of the laboratories. The control values are above the highest reference limit for six of the laboratories. Regarding immunological methods there should be no serious problems concerning analytical specificity of the methods and with an untreated frozen liquid pool the problems are negligible as also indicated by the constancy of the control results. How the reference intervals have been estimated or chosen by the different laboratories has not been investigated - but more than half of the laboratories have serious problems with their reference intervals.

\section{S-Transferrin}

Reference Intervals in Denmark (June 1990)

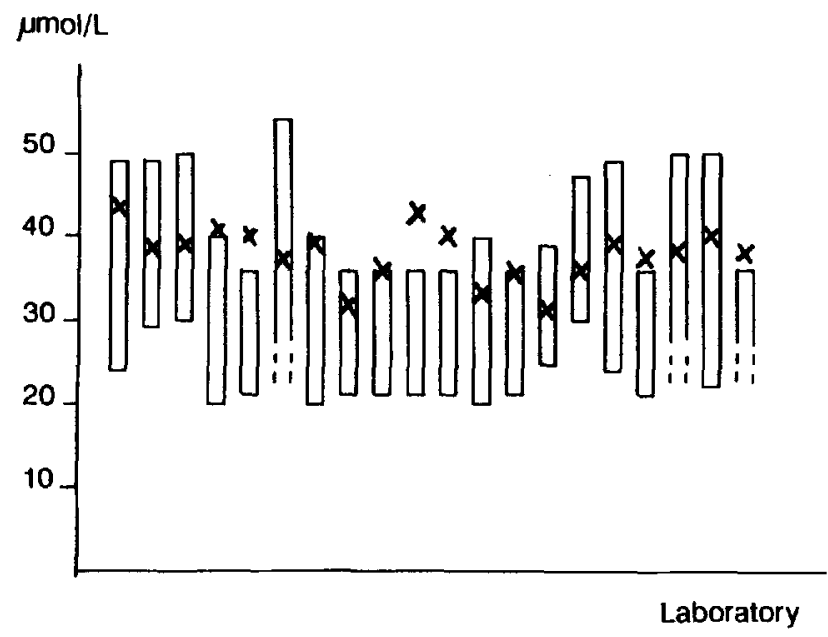

Fig. 7.2.1.2. Comparison of reference intervals and results from an external control survey in Denmark in 1990 for S-Transferrin. For each laboratory the reference interval is shown as a bar and the control result is indicated by a cross.

The two examples clearly demonstrate the problems related to reference intervals for plasma proteins and indicate the need for establishing better reference intervals. One of the presumptions for this is a general and reliable standardization with calibrators and concentration values traceable to a common reference preparation. With the IFCC/CAP/BCR 470 this is now possible - and when we have a general basis for calibration it seems logical to establish common reference intervals for populations homogeneous for the quantities - and to control the analytical quality according to the accept limits given $(4,5)$. 


\subsubsection{Collection of Reference Serum Samples and Measurements}

\section{Presumptions for Establishing Common Reference Intervals}

- The biology must be common

i.e. the population must be homogeneous for the quantity.

- The sampling conditions must be standardized.

- The analytical quality must be acceptable within the quality specifications, i.e. traceable to the same common reference preparation, and with negligible unspecificity.

- The interpretation of reference values must be the same.

\section{Reference Individuals}

Assuming that the population of adults born in Denmark and still living in Denmark is homogeneous for the nine plasma proteins [ S-Prealbumin(Transthyretin), S-Albumin, S-Orosomucoid ( $\alpha_{1}$-Acid Glycoprotein), S- $\alpha_{1}$-Antitrypsin ( $\alpha_{1}$-Trypsin Inhibitor), S-Haptoglobin, S-Transferrin, S-IgA, S-IgG, and S-IgM ] the selection of reference subjects is based on this population using the following additional criteria:

- Hospital personnel and their relatives.

- 20 hospital laboratories from the whole country.

(The population in Denmark is considered homogeneous for the proteins as the area is restricted and the population is well mixed - with only moderate influx of foreigners until 20 years ago).

- State of health: The person must feel healthy

No chronic disease

No intake of regular medications

(insulin, penicillin, antihypertensiva etc.)

- Excluded are blood donors

- Restrictions 24 hours before blood collection:

No exhausting exercise

Usual intake of food

Not more than $12 \mathrm{~g}$ of alcohol (approx. one drink). 
In each of the 20 laboratories blood was collected from a number of persons according to the scheme:

$\begin{array}{cccc}\text { Age Group } & \text { Men } & \text { Women (-E) } & \text { Women }(+E) \\ 18-20 & 2 & 2 & 2 \\ 21-30 & 2 & 2 & 2 \\ 31-40 & 2 & 2 & 2 \\ 41-50 & 2 & 2 & 2 \\ 51-60 & 2 & 2 & \\ 61-70 & 2 & 2 & \\ 71-80 & 2 & 2 & \end{array}$

Blood sampling was performed according to the individual laboratory's routine:

- Sitting position 15 min before and during sampling

- Sampling from arm vein (minimum stasis) in vacuum tubes (if possible)

- Sampling volume for serum approx. $40 \mathrm{~mL}$ (without stabilizers) and for blood to Sedimentation Rate measurements approx. $5 \mathrm{~mL}$ (with citrate)

The Sedimentation Rate was performed locally within few hours.

Handling of blood/serum for protein determinations:

- Coagulation for about one hour at room temperature

- Centrifugation at $1500 * \mathrm{~g}$ for $15 \mathrm{~min}$.

- Pipetting of serum and mixing

- New centrifugation at $1500 * g$ for $15 \mathrm{~min}$.

- Mixing of serum

- Distribution of serum in 8 cryotubes

- Freezing (at $-80^{\circ} \mathrm{C}$ if possible)

Storage and transport:

- 4 tubes sent to the laboratory measuring all samples

- 2 tubes kept in reserve (stored locally)

- 2 tubes for local measurements

\section{Rule out criteria:}

- It is well known, that a number of conditions influence the concentrations of plasma proteins. Some are considered serious, e.g. diseases especially acute phase reactions. Known diseases are excluded as indicated above, whereas, others are unnoticed such as minor infections in an early stage - or even weeks after infections. These conditions are not recognized but are sought to be disclosed by 
measuring Sedimentation Rate and S-CRP and by excluding results from individuals with elevated values of these tests. Further, the presence of $\mathbf{M}^{\prime}$ Components will influence the immunoglobulin concentrations, so results from individuals with detectable M-Components are excluded accordingly. The criteria for excluding were S-CRP above $10 \mathrm{mg} / \mathrm{L}$, Sedimentation Rate according to chapter 7.5, and $\mathrm{M}$-Components if a distinct band is detected in the $\beta$ - $\gamma$-area by agarose electrophoresis. Fortunately, the number of rejections were small ( $7 \%$ ) and none of the values gave reason to assume a serious disease. Blood donors are excluded due to the risk of abnormal values of S-Transferrin and S-Haptoglobin.

- Women using estrogens for anti-conception have been included as a special group.

Measurements of all samples were performed on a Cobas Fara (Roche) according to the method recommendations from DAKO and with antisera from DAKO using the Nordic Calibrator (all values are traceable to IFCC/CAP/BCR 470). Each result is calculated from the mean of two measurements performed in separate runs. The controls (A, B, and C) from the Nordic Protein Project were used according to the Westgard-rules (Mean and range rules).

Measurements of S-C-Reactive Protein, S-CRP, were performed by Søren BlirupJensen and Per Just Svendsen, DAKO.

Electrophoreses for disclosing M-Components were performed at the Department of Clinical Chemistry, Hjørring Hospital.

The data base was designed for the purpose and calculations of mean, standard deviations, skewness and kurtosis for concentrations and log-values as well as for percentiles for the different combinations of individuals were performed by the computer programme SPSS.

\section{Reference individuals}

It was the goal to collect $20 * 36=720$ reference samples. But only 553 samples were received from the 20 laboratories. 37 of these had to be excluded due to the rule out criteria (high Sedimentation Rate, high S-CRP, and the presence of M-Components. Two had raised values of both Sedimentation Rate and S-CRP and one had both a Mcomponent and elevated Sedimentation Rate. In total 9 M-Components, 15 elevated SCRP, and 16 elevated Sedimentation Rates were found. 
The total number of included reference individuals are:

\begin{tabular}{ccccc} 
Age Group & Men & & Women (-E) & Women (+E) \\
\cline { 1 - 2 } $18-20$ & 31 & 26 & 24 \\
$21-30$ & 37 & 38 & 34 \\
$31-40$ & 33 & 38 & 26 \\
$41-50$ & 36 & 40 & 24 \\
$51-60$ & 30 & 34 & \\
$61-70$ & 20 & 27 & \\
$71-80$ & 14 & 7 &
\end{tabular}

The number of individuals in the older age groups are small and so less representative in the calculations. The general approach for lumping the groups has been Men below 50 years of age (137), Women below 50 (not using estrogen) (142), Women below 50 (using estrogen) (108), Men above 50 (64), and Women above 50 (68). The criteria for selection of reference individuals are in close agreement with the recommendations from the Nordic recommendations for production of reference values from adults (1), whereas the rule out criteria are specific for estimation of reference intervals for plasma proteins.

\subsubsection{Biology and Reference Intervals for Eight Proteins}

For each of eight proteins the biological variations according to age and sex as well as to the effect of estrogens are illustrated by their means and the 0.90 (90\%) confidence intervals, CI. The ordinates are logarithmic as most of the distributions are close to log-Gaussian. Further, the distributions of the concentrations are shown in probit plots according to the subgroupings and finally the 0.95 interfractile reference intervals are given. The plasma protein $\alpha_{1}$-Antitrypsin is described separately in chapter 7.3 .

\section{S-Prealbumin (S-Transthyretin).}

The biological distributions according to age and sex are given in Fig. 7.2.3.1.A. The figure shows that the group of women below 50 years of age not using estrogens has lower values. The remaining mean-values seem to describe a curve with increasing values up to about 40 years, and then descending again. It is possible to choose separate reference intervals for each subgroup but, the degrees of freedom are small and according to the criteria (section 7.1) it is not reasonable to do so. 


\section{S-Prealbumin}

\section{Concentration}

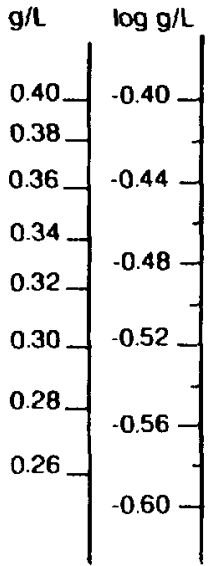

Fig. 7.2.3.1.A.

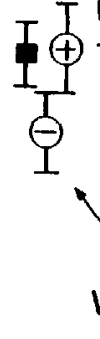

$\Gamma$

18-20 21-30 31-40 41-50 51-60 61-70 71-80 Age Groups

Women (-)
I Men

I women

Mean and $0.90 \mathrm{Cl}$

Mean concentrations with $90 \%$ CI for each subgroup shown on a log-scale in relation to age for S-Prealbumin (S-Transthyretin).

\section{S-Prealbumin}

Cumulated percentage frequency

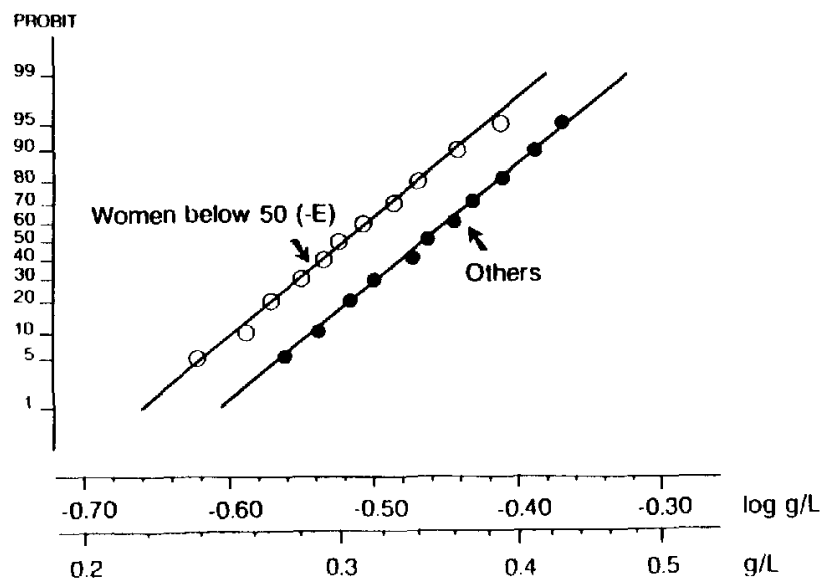

Fig. 7.2.3.1.B.

Probit-plot with log-abscissa showing S-Prealbumin (S-Transthyretin) distributions for women not using estrogens and the remaining group (all others). 
In Fig. 7.2.3.1.B the two distributions are clearly straight lines indicating log-Graussian distributions close to be parallel, accordingly the reference intervals are given as 0.23 to $0.39 \mathrm{~g} / \mathrm{L}$ for women below 50 years of age not using estrogens and 0.26 to $0.45 \mathrm{~g} / \mathrm{L}$ for the remaining adults.

\section{S-Albumin.}

The biological distributions according to age and sex are given in Fig. 7.2.3.2.A. The figure shows that the group of women below 50 years of age using estrogens differ with lower values from the other individuals below 50 years. The mean values of the remaining groups seem to descend with increasing age. It is possible to choose separate reference intervals for each subgroup but, we have chosen to cut at the age of 50 and combining the women below 50 using estrogens with the individuals above 50 years of age.

\section{Concentration}

$g / L \quad \log g / L$
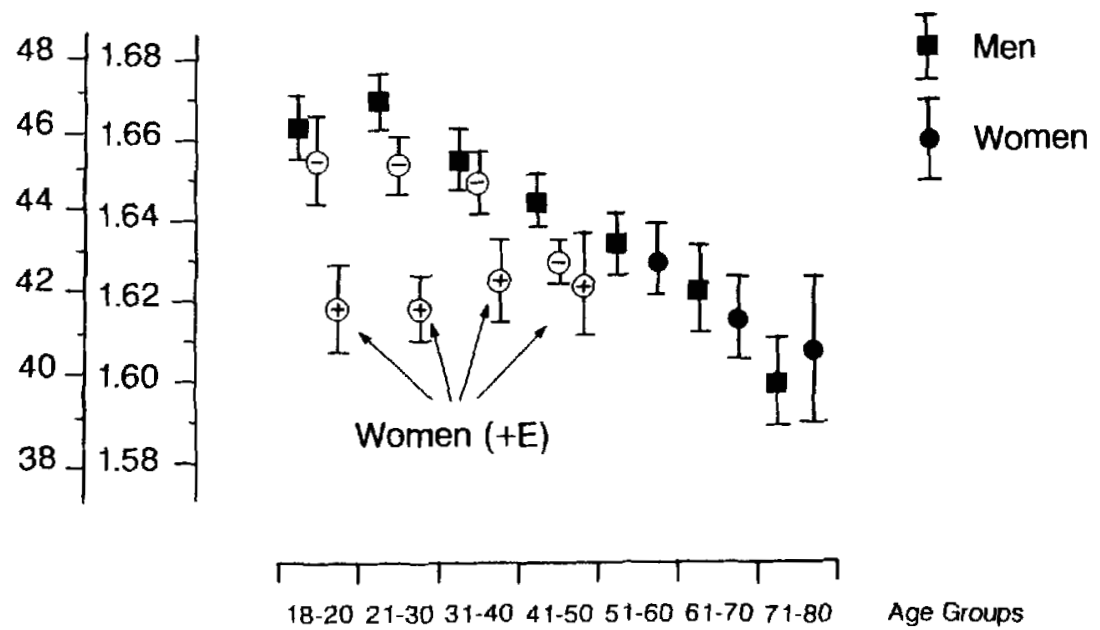

Fig. 7.2.3.2.A.

Mean concentrations with $90 \%$ CI for each subgroup shown on a log-scale in relation to age for S-Albumin.

In Fig. 7.2.3.2.B the two distributions are clearly straight lines indicating log-Gaussian distributions close to be parallel, accordingly the reference intervals are given as 39.6 to $51.1 \mathrm{~g} / \mathrm{L}$ for men below 50 and women below 50 years of age not using estrogens and 36.6 to $48.2 \mathrm{~g} / \mathrm{L}$ for the remaining adults. 


\section{S-Albumin}

Cumulated Percentage Frequency

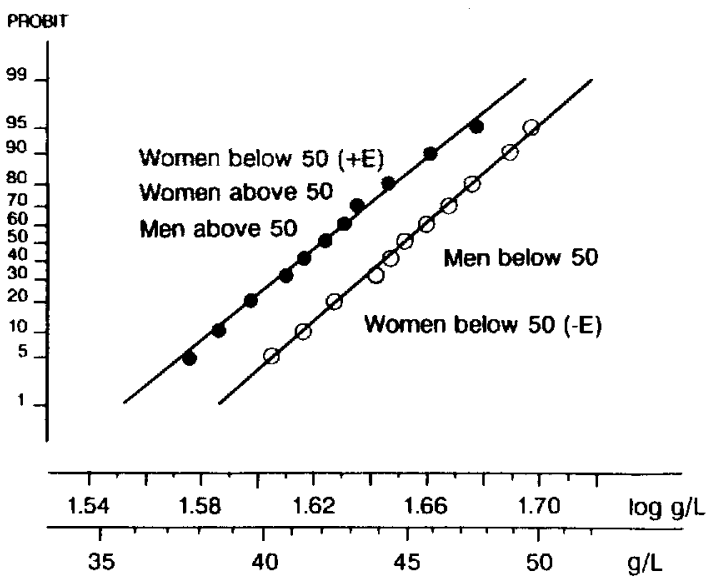

Fig. 7.2.3.2.B.

Probit-plot with log-abscissa showing S-Albumin distributions for men and women not using estrogens, both below 50 years of age and the remaining group (all others).

\section{S-Orosomucoid (S- $\alpha_{1}$-Acid Glycoprotein)}

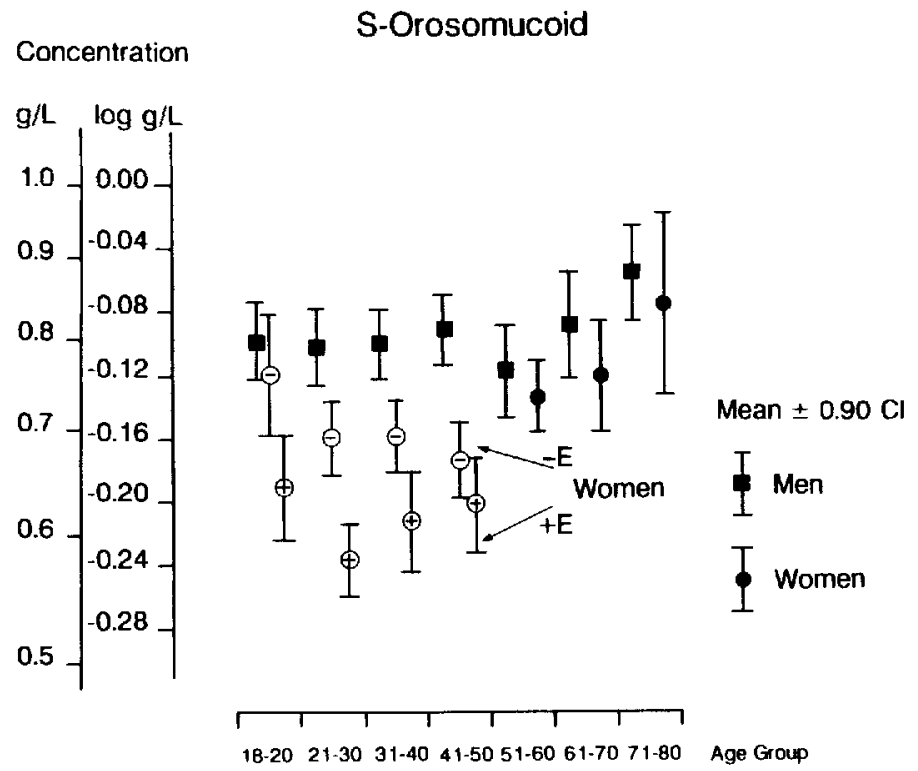

Fig. 7.2.3.3.A.

Mean concentrations with $90 \%$ CI for each subgroup shown on a log-scale in relation to age for $S$-Orosomucoid. 
The biological distributions according to age and sex are given in Fig. 7.2.3.3.A. The figure shows that the women below 50 years of age separate into two groups according to the use of estrogens, both groups are clearly lower than the remaining groups. The men show constancy and women above 50 are at the same level. It is possible to choose separate reference intervals for each subgroup of women below 50 years of age as illustrated in Fig. 7.2.3.3.B.

\section{S-Orosomucoid}

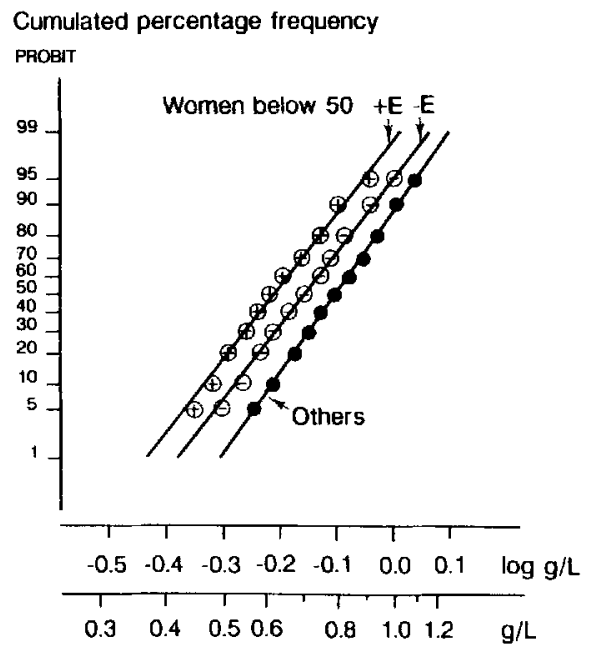

Fig. 7.2.3.3.B.

Probit-plot with log-abscissa showing for S-Orosomucoid the distributions for women not using estrogens, for women using estrogens both below 50 years of age and the remaining group (all others).

In Fig. 7.2.3.3.B two of the distributions are clearly straight lines indicating logGaussian distributions, whereas, the distribution for women using estrogens might be a little more skew. In order to investigate whether it is reasonable to separate between the women below 50 with and without estrogens the 0.90 confidence intervals for the percentiles (with 107 degrees of freedom) are shown as the hatched area around the line for women using estrogens in Fig. 7.2.3.3.C. There is no overlap with the group not using estrogens, which in turn also differ from the remaining group as the degrees of freedom are 141 (not shown in the figure).

All three lines are close to be parallel so, the reference intervals are given as 0.40 to $0.95 \mathrm{~g} / \mathrm{L}$ for women below 50 years of age using estrogens, 0.45 to $1.08 \mathrm{~g} / \mathrm{L}$ for women below 50 not using estrogens and 0.54 to $1.17 \mathrm{~g} / \mathrm{L}$ for the remaining adults. $\mathrm{S}$ Orosomucoid is the only protein where the reference intervals are given for three separate groups. 


\section{S-Orosomucoid}

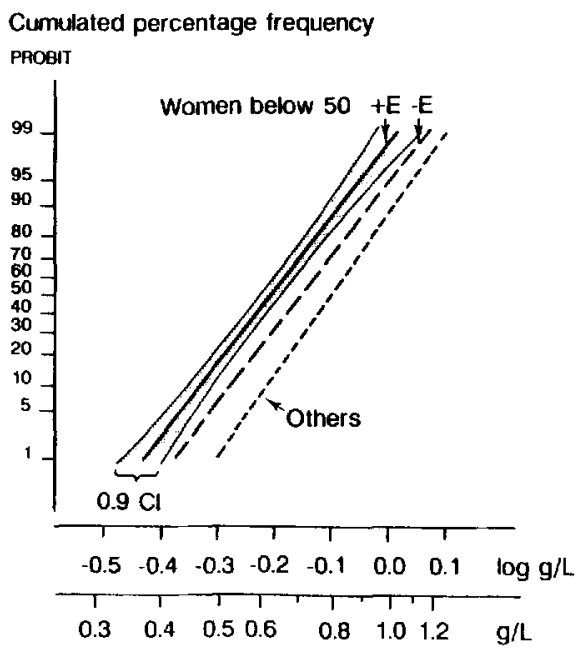

Fig. 7.2.3.3.C.

Probit-plot with log-abscissa for S-Orosomucoid showing the same distributions as in Fig 7.2.3.3.B, but with 0.90 CI for percentiles for 107 degrees of freedom around the lin for women using estrogens.

\section{S-Haptoglobin}

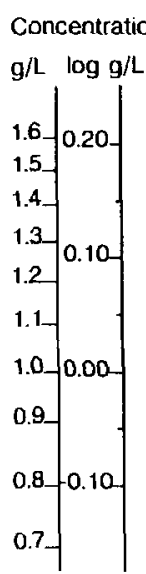

\section{S-Haptoglobin}
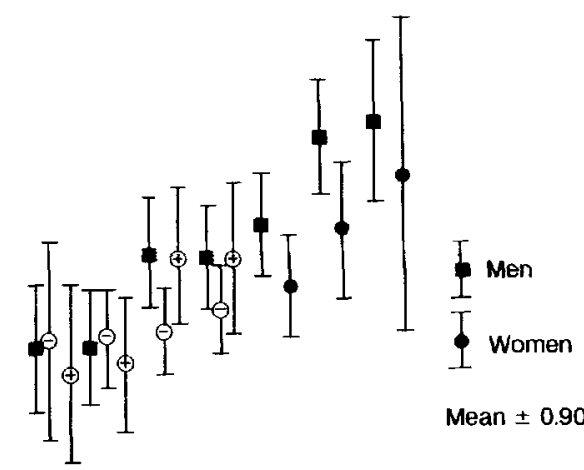

Mean $\pm 0.90 \mathrm{Cl}$

Fig. 7.2.3.4.A.

Mean concentrations with $90 \%$ CI for each subgroup shown on a log-scale in relation to age for S-Haptoglobin. 
The biological distributions for S-Haptoglobin according to age and sex are given in Fig. 7.2.3.4.A. The figure shows age to be the dominating factor as the concentrations are increasing with age. Accordingly, the values are separated into two groups, one below 50 and one above 50 years of age.

\section{S-Haptoglobin}

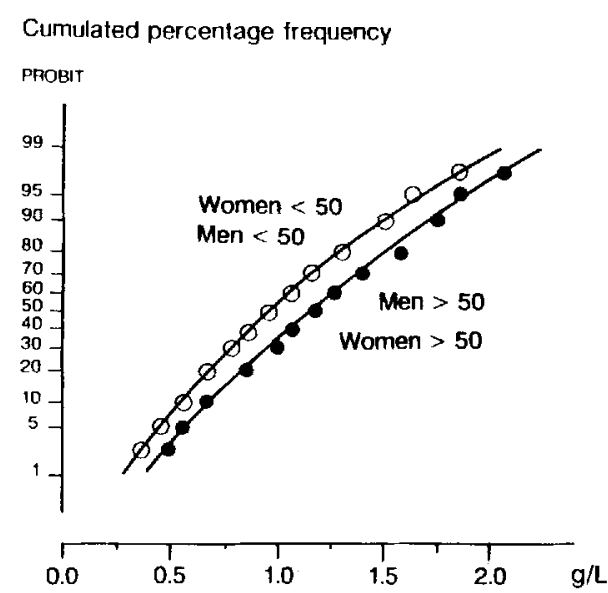

Fig. 7.2.3.4.B.

Probit-plot with linear abscissa showing for S-Haptoglobin the distributions for individuals below 50 and individuals above 50 years of age.

The two groups are neither log-Gaussian, nor Gaussian, and the distributions are therefore shown with linear abscissa in Fig. 7.2.3.4.B. Both distributions are curved but the difference seems to be rather constant at all levels. This difference does not seem important due to the broad intervals but, we have decided to keep two separate intervals. For individuals below 50 the reference interval is 0.35 to $1.85 \mathrm{~g} / \mathrm{L}$ and above 50 years of age it is from 0.47 to $2.05 \mathrm{~g} / \mathrm{L}$.

\section{S-Transferrin}

The biological variations and the distributions are described in section 7.1 in which STransferrin is used as an example for the presentations and main problems. Reference interval for women below 50 years of age using estrogens is 2.25 to $3.85 \mathrm{~g} / \mathrm{L}$ and for the remaining group 1.94 to $3.26 \mathrm{~g} / \mathrm{L}$.

\section{S-IgA}

S-IgA resemble S-Haptoglobin with values increasing with age and no difference due to sex and to estrogen as seen from Fig. 7.2.3.5.A. 


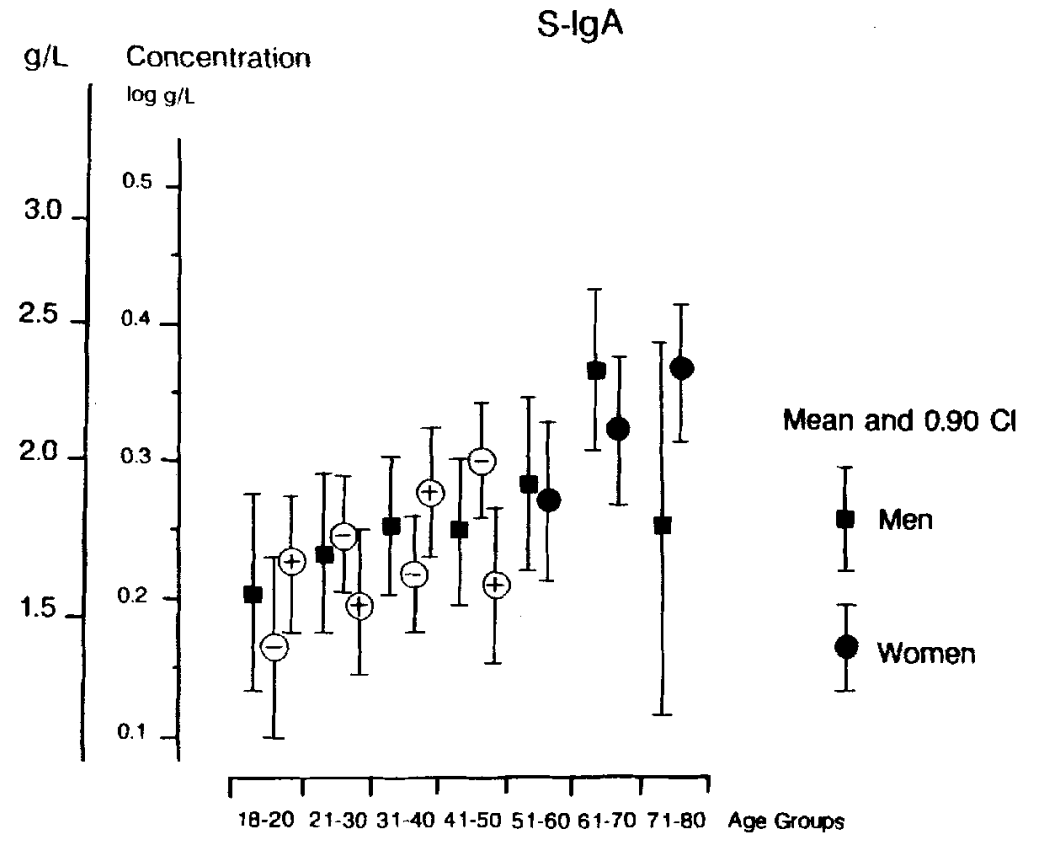

Fig. 7.2.3.5.A. Mean concentrations with $90 \%$ CI for each subgroup shown on a log-scale in relation to age for $S-\operatorname{Ig} A$.

\section{S-IgA}

Cumulated Percentage Frequency

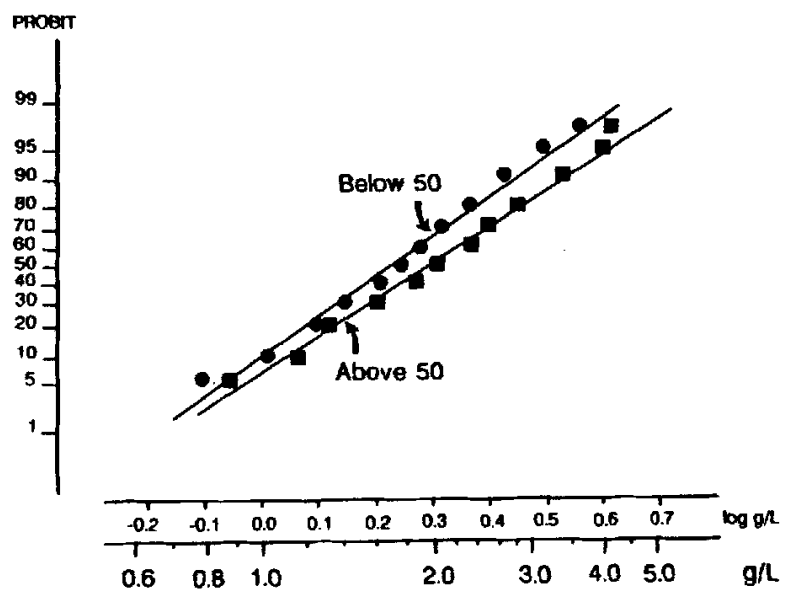

Fig. 7.2.3.5.B.

Probit-plot with log-abscissa showing S-IgA distributions for the individuals below 50 and the individuals above 50 years of age. 
In the probit-plot (Fig. 7.2.3.5.B) the two distributions are not two straight lines on the $\log$-scale. But, by adding $0.50 \mathrm{~g} / \mathrm{L}$ to all values (5) the distributions seem to be nearly log-Gaussian as seen in Fig. 7.2.3.5.C. The highest values, however, seem to diverge from the straight line so the calculation of reference intervals has been performed according to non-parametric estimations of the $2 \frac{1}{2}$ and $971 / 2$ percentiles.

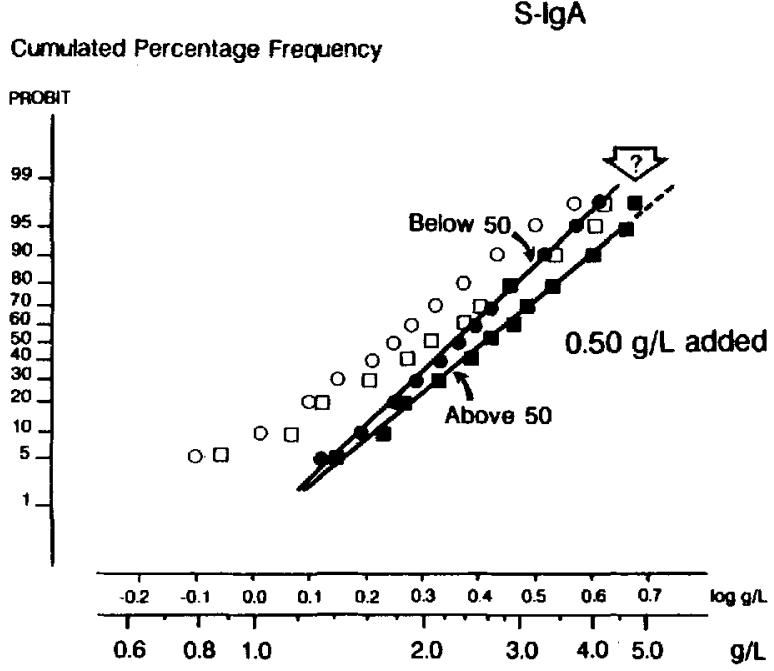

Fig. 7.2.3.5.C.

Probit-plot with log-abscissa showing the same distributions for S-IgA as in Fig 7.2.3.3.B, and after addition of $0.5 \mathrm{~g} / \mathrm{L}$ to all values. The question mark indicates the deviation from a straight line.

The reference intervals according to non-parametric estimations for individuals below 50 are 0.70 to $3.65 \mathrm{~g} / \mathrm{L}$ and for individuals above 50 years of age 0.70 to $4.30 \mathrm{~g} / \mathrm{L}$.

\section{S-IgG}

The biological distributions according to age and sex are given in Fig. 7.2.3.6.A. The figure shows that the group of women below 50 years of age differ with higher values. The remaining mean-values do not describe a clear pattern and it might be possible to separate the women below 50 in two groups according to estrogen. We have decided, however, to distinguish between women below 50 and the remaining group as illustrated in Fig 7.2.3.6.B. 
Concentration

S-lgG

$\mathrm{g} / \mathrm{L} \quad \log \mathrm{g} / \mathrm{L}$

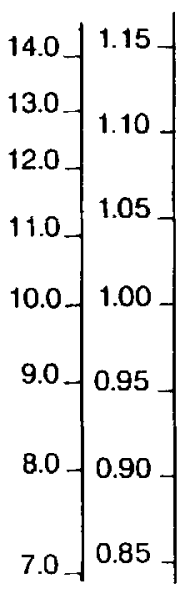

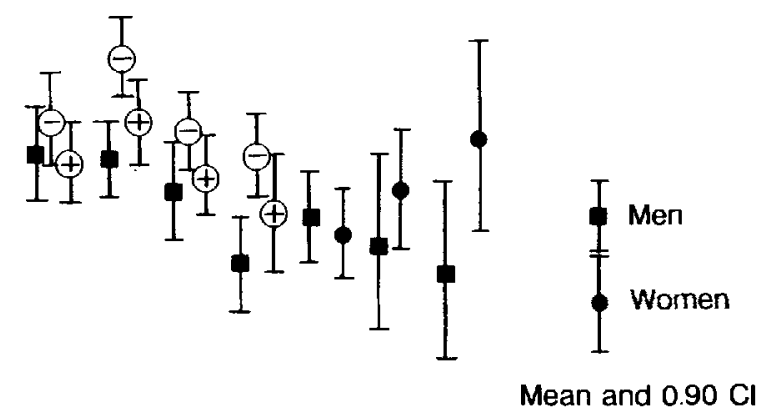

Fig. 7.2.3.6.A.

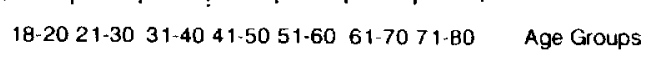

Mean concentrations with $90 \%$ CI for each subgroup shown on a log-scale in relation to age for $S-\operatorname{Ig} G$.

\section{S-lgG}

Cumulated percentage frequency

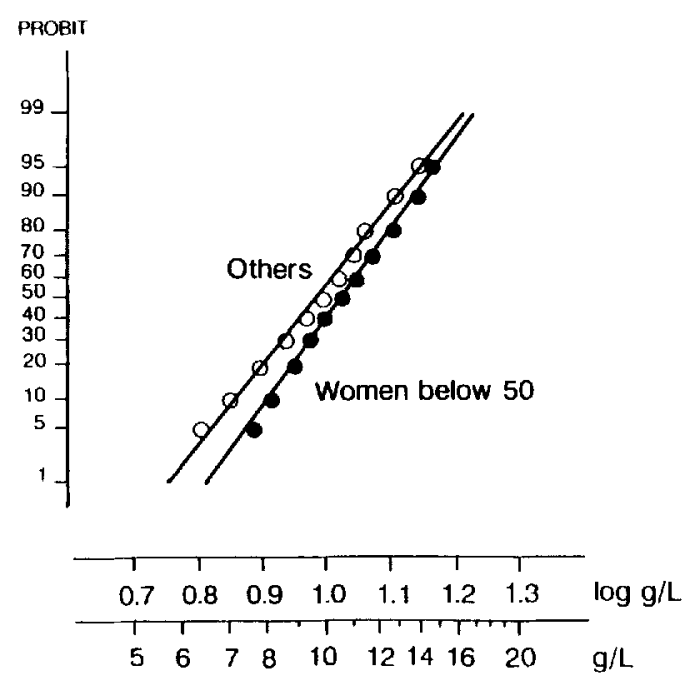

Fig. 7.2.3.6.B.

Probit-plot with log-abscissa showing S-IgG distributions for women below 50 years of age and the remaining group (all others). 
In Fig. 7.2.3.6. B the two distributions are close to straight lines (with minor deviations in both directions) indicating that they are close to log-Gaussian.

The reference intervals for S-IgG are given as 6.9 to $15.7 \mathrm{~g} / \mathrm{L}$ for women below 50 years of age and 6.1 to $14.9 \mathrm{~g} / \mathrm{L}$ for the remaining adults.

\section{S-IgM}

The biological distributions according to age and sex are given in Fig. 7.2.3.7.A. The figure shows that the group of women below 50 years of age differs with higher values. The remaining mean-values seem to be constant with age.

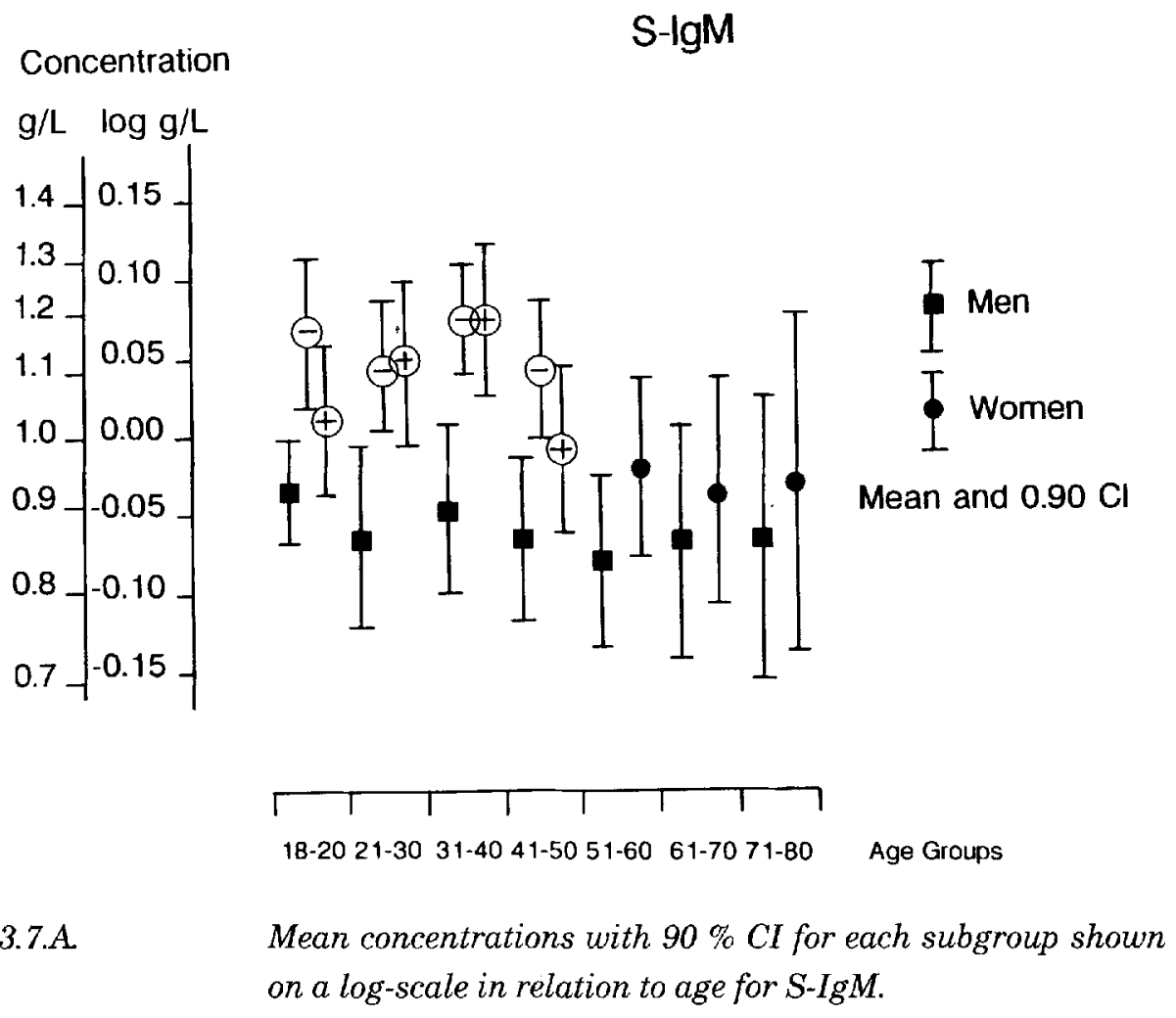

In Fig. 7.2.3.7.B the two distributions are close to straight lines indicating logGaussian distributions.

The reference intervals for S-IgM are given as 0.55 to $2.30 \mathrm{~g} / \mathrm{L}$ for women below 50 years of age and 0.39 to $2.08 \mathrm{~g} / \mathrm{L}$ for the remaining adults. 


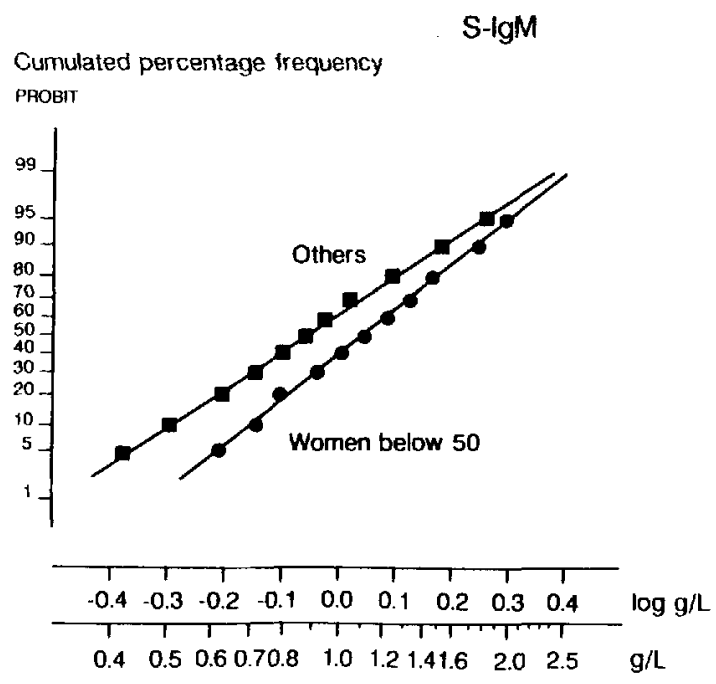

Fig. 7.2.3.7.B. Probit-plot with log-abscissa showing S-IgM distributions for women below 50 years of age and the remaining group (all others).

\subsubsection{Distributions of Values from the Individuals not Included due to the Rule Out Criteria}

For the estimation of reference intervals 37 individuals were ruled out due to high Sedimentation Rate, high S-CRP or the presence of M-Component. Among the 16 individuals with elevated Sedimentation Rate two had elevated S-CRP as well and one had an M-Component. 15 had elevated S-CRP (two of these with high Sedimentation Rate) and 9 disclosed an M-Component (one also with a high Sedimentation Rate). As the three conditions are expected to be related to changes in plasma proteins it is interesting to investigate whether the results are different from the reference individuals.

In the following the distributions are shown on probit-scales in relation to the reference distributions. The number of values, and thereby, the degrees of freedom and the reliability is not impressive but as the majority of the values show a tendency, some tentative conclusions may be made.

For S-Prealbumin (Fig. 7.2.4.1) there is a clear tendency to lower values when S-CRP is elevated, whereas, the elevated Sedimentation Rate and the presence of $M$ Components seem to have negligible effects. 


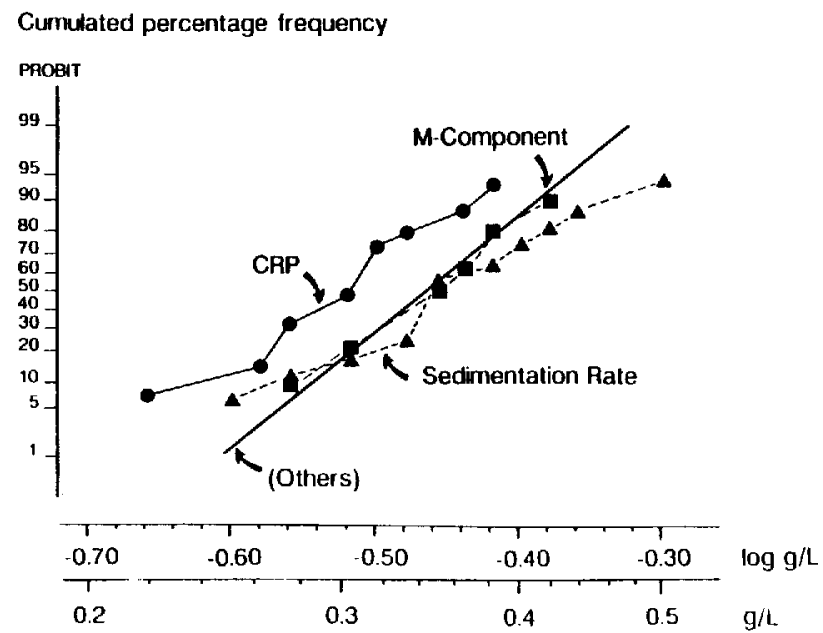

Fig. 7.2.4.1. Distributions of concentration values for S-Prealbumin from the excluded individuals compared to the group 'Others'.

One woman below 50 and not using estrogens but disclosing high values of both Sedimentation Rate and S-CRP is excluded, all the others are comparable to the distribution of others.

\section{S-Albumin}

Cumulated Percientage Frequency

PROBIT

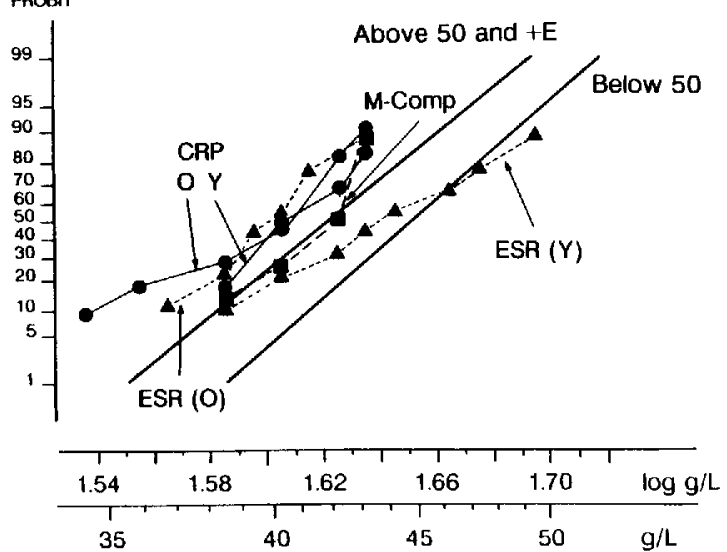

Fig. 7.2.4.2. Distributions of concentration values for S-Albumin from the excluded individuals. 
For S-Albumin (Fig. 7.2.4.2) distributions for individuals below and above 50 are shown for the groups with high Sedimentation Rate ( 8 in each group) as well as for high S-CRP ( 5 below 50 (not using estrogens) and 10 in the remaining group). Two individuals are excluded from the group of M-Component, reducing the number to 7 , which should be compared to the lower distribution. There is a tendency to lower values in all groups but, only for the group of men and women (not using estrogens) below 50 years of age there is a more convincing decrease.

\section{S-Orosomucoid}

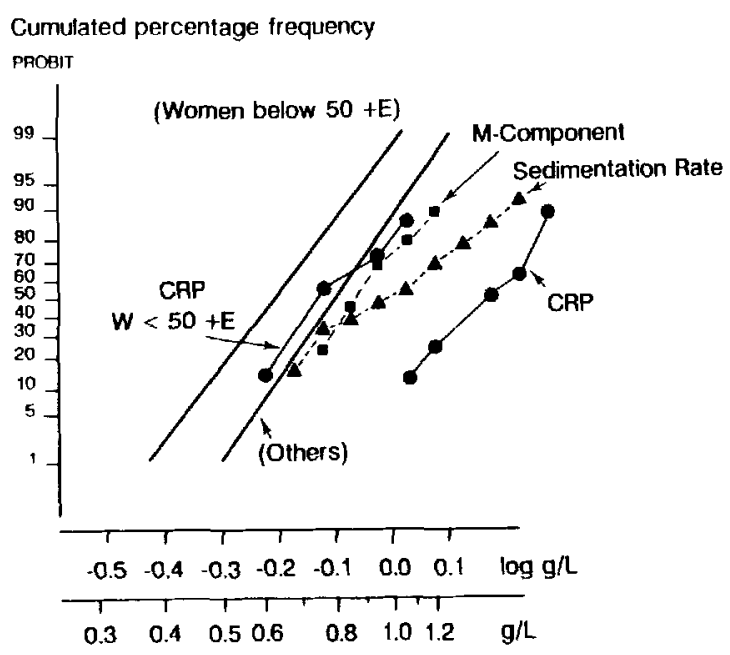

Fig. 7.2.4.3. Distributions of concentration values for S-Orosomucoid from the excluded individuals.

For S-Orosomucoid (Fig. 7.2.4.3) Women below 50 are excluded from the groups with M-Components and elevated Sedimentation Rate, leaving 8 and 12 individuals in the two groups, respectively. The group with elevated S-CRP is separated in women using estrogens $(n=6)$ and men and women above $50(n=7)$.

M-Components reveal no effect but elevated Sedimentation Rate indicates increased values and high S-CRP is accompanied by increased S-Orosomucid values, most distinct for women below 50 using estrogens.

For S-Haptoglobin (Fig. 7.2.4.4) there is a slight tendency for higher values in all groups, but the distributions are broader than the distributions for the reference values. 


\section{S-Haptoglobin}

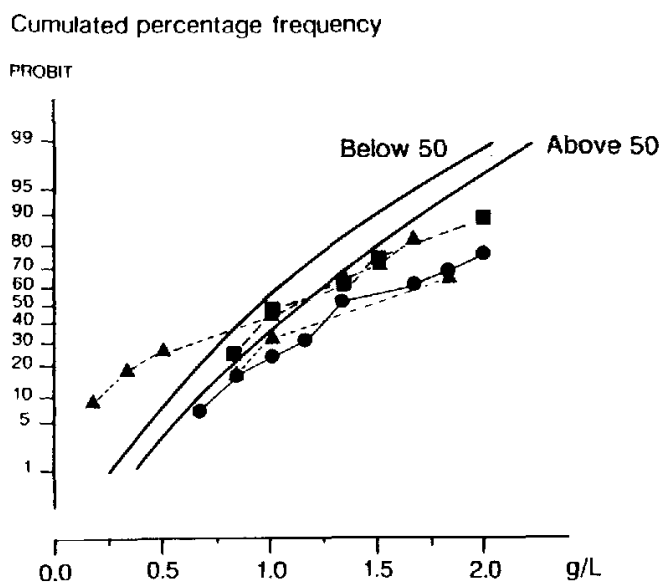

Fig. 7.2.4.4. Distributions of concentration values for S-Haptoglobin from the excluded individuals.

S-Transferrin (see chapter 7.1) resembles S-Albumin with slightly decreased values in all groups, less pronounced for Sedimentation Rate. M-Component-group with $\mathbf{n}=8$ and Sedimentation Rate $n=13$ (women using estrogens excluded), and S-CRP $\mathbf{n}=7$ for women using estrogens and $n=8$ for the remaining group.

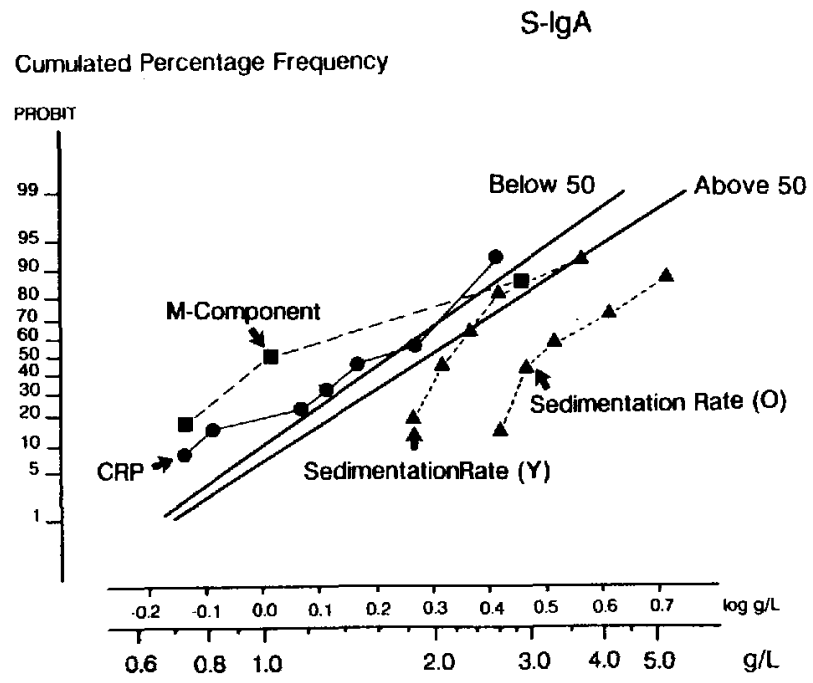

Fig. 7.2.4.5. Distributions of concentration values for S-IgA from the excluded individuals. 
Concerning S-IgA (Fig. 7.2.4.5) one individual had a M-Component of class IgA (and an additional of type $\mathrm{G}$ ). The M-Component group consisted of $\mathrm{n}=6$ (excluding ' 2 individuals below 50). For S-CRP 12 individuals below 50 were included, and the Sedimentation Rate- group separated in 10 below 50 and 6 above 50. For the MComponent group there was a tendency to lower values and for the Sedimentation Rate groups higher S-IgA values were obtained.

\section{S-IgG}

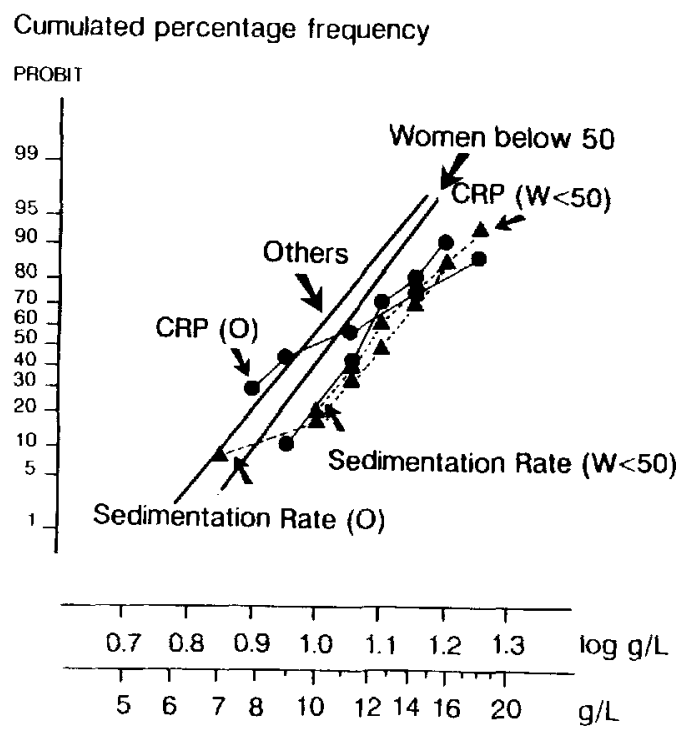

Fig. 7.2.4.6. Distributions of concentration values for S-IgG from the excluded individuals.

Seven of the nine M-Components were of class IgG, so there is no distribution for MComponents in Fig. 7.2.4.6. Both the Sedimentation Rate group and the S-CRP group were separated into two: S-CRP: women below $50 \mathrm{n}=9$ and the remaining $\mathrm{n}=6$, and for Sedimentation Rate for the same groups $n=4$ and $n=12$, respectively.

The values of S-IgG for all groups seem to be slightly increased.

Two M-Components of type IgM were found in the material and the corresponding SIgM values are excluded. The separation into subgroups is the same as for S-IgG.

The distributions as shown in Fig. 7.2.4.7, are close to the the reference groups, except from a few higher values and a tendency to higher values of S-IgM in individuals with elevated Sedimentation Rate. 


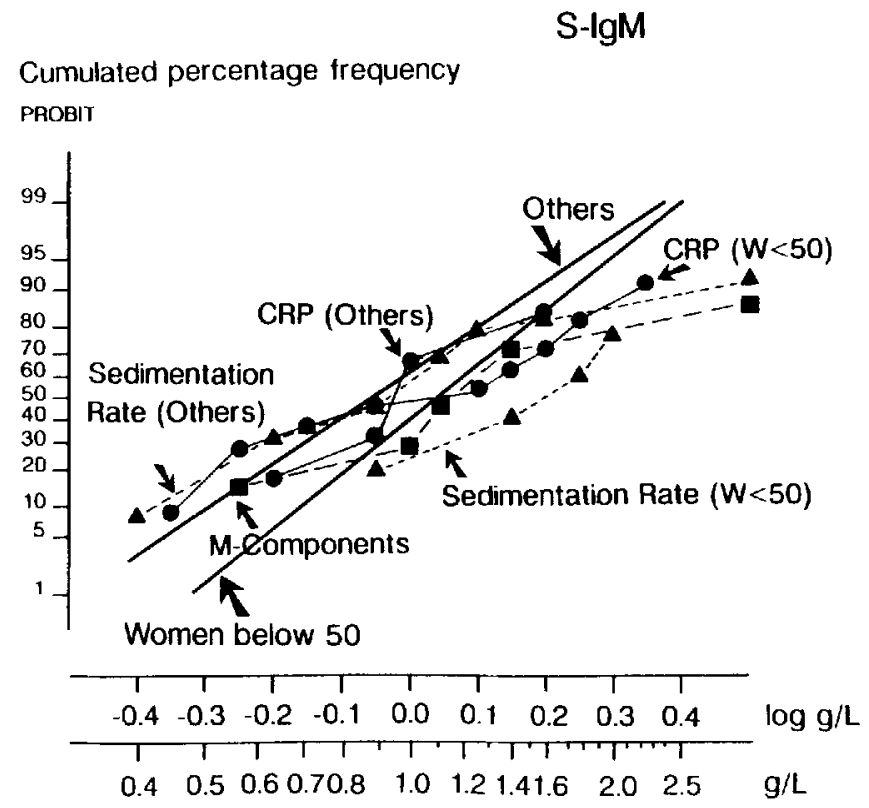

Fig. 7.2.4.7. Distributions of concentration values for S-IgM from the excluded individuals.

As a general conclusion based on the figures showing the distributions of values from the individuals who were ruled out, it is confirmed that relationships exist between the concentrations of the eight plasma proteins and the two acute phase indicators as well as minor M-Components. This implies that the same rule out criteria should be used when plasma protein reference intervals for other ethnic groups are considered.

\section{Discussion}

The estimations of reference intervals were planned in order to supplement the calibration and control projects by providing the reference intervals needed for the total quality concept, i.e. 1) common reference intervals, 2) analytical quality specifications for sharing these common reference intervals, 3) creation of analytical quality needed, and 4) control of analytical quality according to the purpose. The sample size of reference individuals was, therefore, designed to approx. 800 (4), actually 720 individuals. The obtained number, however, was 516 , which is a considerable number, but less than needed for the ideal situation. Further, only minor differences between the groups were expected, except from the the estrogen effects. The actual disclosed - and unexpected - differences, however, varied from protein to protein and thus reduced the certainty of the distributions of each subgroup. 
Therefore, the conclusions concerning some of the estimated reference intervals are less certain than expected from the design. A new and better design could be described based on the results obtained here, but even with the limitations of the material, the presented common reference intervals are better than any solution with individual reference intervals for each laboratory presupposing the use of a common calibrator.

\section{Acknowledgements}

We want to thank all the persons who have contributed to this work, volunteers who gave blood to the investigation and the 20 Danish laboratories involved in collecting the blood samples and doing extra analytical work. We are in debt of gratitude to Ane Richter, who performed the registrations and calculations of the many results. Further we are grateful to Søren Blirup and Per Just Svendsen, who performed the measurements of S-CRP and to DAKO for the antisera used in all the determinations.

\section{References}

1. Alström T, Gräsbeck R, Lindblad B, Solberg HE, Winkel P, Viinikka L. Establishing reference values from adults: recommendation on procedures for the preparation of individuals, collection of blood, and handling and storage of specimens. Scand J Clin Lab Invest 1993;53:649-52.

2. Djurhuus MS, Rohold A, Vadstrup S, Hyltoft Petersen P, Uldall A. Reference intervals based on hospitalized 'healthy' patients and medical students in relation to analytical bias for serum potassium. Scand J Clin Lab Invest 1992;52:305-12.

3. Frølich A, Nielsen BF, Nielsen J, Conradsen K, McNair P. Do local hospitals meet the analytical goals for the use of common reference intervals? Scand J Clin Lab Invest 1994;54:169-76.

4. Gowans EMS, Hyltoft Petersen P, Blaabjerg O, Horder M, Analytical Goals for the Acceptance of Common Reference Intervals for Laboratories Throughout a Geographical Area. Scand J Clin Lab Invest 1988;48:757-64.

5. Hyltoft Petersen P, Gowans EMS, Blaabjerg O, Hørder M, Analytical Goals for Estimation of nonGaussian Reference Intervals. Scand J Clin Lab Invest 1889;49:727-37. 